\title{
STABILITY OF ZERO SOLUTION OF SYSTEM WITH SWITCHES CONSISTING OF LINEAR SUBSYSTEMS
}

\author{
D. Khusainov ${ }^{1}$, A. Bychkov ${ }^{2}$, A. Sirenko ${ }^{3}$ \\ ${ }^{1}$ Faculty of Computer Science and Cybernetics, Taras Shevchenko Kiev National University, \\ Kiev, Ukraine, E-mail: d.y.khusainov@gmail.com \\ ${ }^{2}$ The Faculty of Information Technology, Taras Shevchenko Kiev National University, Kiev, \\ Ukraine, E-mail: bos.knu@gmail.com \\ ${ }^{3}$ Institute Of International Relations, Taras Shevchenko Kiev National University, Kiev, \\ Ukraine, E-mail: sandrew@online.ua
}

\section{УСТОЙЧИВОСТЬ НУЛЕВОГО РЕШЕНИЯ СИСТЕМЫ С ПЕРЕКЛЮЧЕНИЯМИ, СОСТОЯЩЕЙ ИЗ ЛИНЕЙНЫХ ПОДСИСТЕМ}

\author{
Д. Я. Хусаинов ${ }^{1}$, А. С. Бычков ${ }^{2}$, А. С. Сиренко ${ }^{3}$ \\ ${ }^{1}$ Факультет компьютерных наук и кибернетики, Киевский национальный университет \\ имени Тараса Шевченко, Киев, Украина, E-mail: d.y.khusainov@gmail.com \\ ${ }^{2}$ Факультет информационных технологий, Киевский национальный университет имени \\ Тараса Шевченко, Киев, Украина, E-mail: bos.knu@gmail.com \\ ${ }^{3}$ Институт международных отношений, Киевский национальный университет имени Та- \\ раса Шевченко, Киев, Украина, E-mail: sandrew@online.ua
}

Abstract. In this paper, discusses the study of the stability of solutions of dynamic systems with switching. Sufficient conditions are obtained for the asymptotic stability of the zero solution of switching systems consisting of linear differential and difference subsystems. It is proved that the existence of a common quadratic Lyapunov function is sufficient for asymptotic stability.

KEYWORDS: differential equations, difference equations, dynamical systems, switching system, Lyapunov function.

АннотАция. В работе рассматриваются вопросы исследования устойчивости решений динамических систем с переключениями. Получены достаточные условия асимптотической устойчивости нулевого решения систем с переключениями, состоящих из линейных дифференциальных и разностных подсистем. Показано, что для асимптотической устойчивости достаточно существования общей квадратичной функции Ляпунова.

КлЮчЕВыЕ СЛОВА: дифференциальные уравнения, разностные уравнения, динамические системы, система с переключениями, функция Ляпунова. 


\section{ВВЕДЕНИЕ}

В настоящее время имеется достаточно много работ, посвященных динамическим системам, состоящим из разнородных подсистем, функционирующих на различных временных промежутках $[2-6,8-10,13-16]$.

Существует много математических моделей исследования таких систем. Среди них можно выделить агрегативные, импульсные, логико-динамические, импульсные, системы с переменной структурой, гибридные и др. Над проблемами построения общей теории таких систем работали такие ученые, как Бусленко Н. П., Глушков В. М., Самойленко А. М., Емельянов С. В., Пнуэли А. и др. Одним из видов математических объектов, которые используются для описания и исследования такого вида динамических систем являются системы с переключениями.

Под системами с переключениями будем понимать системы, которые описываются совокупностью дифференциальных уравнений, функционирующих на конечных промежутках времени, переключениями, которые описаны разностными уравнениями, и снова дифференциальными уравнениями. Таким образом, функционирование системы с переключениями можно описать совокупностью уравнений

$$
\dot{x}(t)=f_{i}(x(t), t), i \in I, x\left(t_{k}+0\right)=g_{k}\left(x\left(t_{k}-0\right), t_{k}-0\right), k \in K, t \geq 0 .
$$

Функции $f_{i}(x(t), t), i \in I$ и $g_{k}(x(t), t), k \in K$ принадлежат заданным классам функций

$$
f_{i}(x(t), t) \in \Phi_{i}, i \in I, g_{k}(x(t), t) \in \Gamma_{k}, k \in K .
$$

удовлетворяют нулевым условиям $f_{i}(0, t) \equiv 0, g_{k}(0, t) \equiv 0, i \in I, k \in K$. т.е. $x(t) \equiv 0$ является решением системы $(1)$, и система с переключениями такова, что ее решения удовлетворяют условиям существования и единственности и непрерывно зависят от начальных условий. Авторы настоящей работы рассматривают два класса систем с переключениями.

\section{1. СИСТЕМЫ С ОПРЕДЕЛЕННЫМИ ПЕРЕКЛЮЧЕНИЯМИ}

Для систем такого вида заданы интервалы переключения, системы дифференциальных уравнений, которые функционируют на этих промежутках и, соответственно, системы разностных уравнений, которые определяют переключения. Системы такого вида назовем системами с определенными переключениями. Даже, если промежутки функционирования подсистем дифференциальных уравнений конечны, а сами подсистемы имеют асимптотически устойчивое положение равновесия, то положение равновесия системы с переключениями в целом может быть неустойчивой $[9,18]$. В этом случае, существенным является выбор моментов переключения («из двух асимптотически устойчивых подсистем можно синтезировать одну неустойчивую систему»). Исследование устойчивости нулевого положения равновесия систем с определенными переключениями проводилось в работах $[11,12]$. Методом исследования был выбран второй метод Ляпунова. Для каждой из подсистем строилась своя функция Ляпунова. Поскольку 
промежутки времени были заданы, а подсистемы известны, то при получении оценок устойчивости и сходимости поверхность уровня каждой последующей функции Ляпунова в моменты переключения охватывала поверхность уровня предыдущей. Таким образом, поверхность уровня последовательности функций Ляпунова содержала внутри себя возмущенную траекторию. И оценки устойчивости можно было делать с использованием оценок поверхностей уровня.

\section{2. СИСТЕМЫ С НЕОПРЕДЕЛЕННЫМИ ПЕРЕКЛЮЧЕНИЯМИ}

Эти системы также описываются подсистемами дифференциальных и разностных уравнений (1). Однако моменты переключений $t=t_{k}$ или неизвестны, или для их определения требуется решать логические уравнения, что не всегда возможно. Кроме того, для соответствующих временных промежутков неизвестна дифференциальная подсистема (предполагается, что подсистемы принадлежат заданным классам). Исследование асимптотической устойчивости нулевого положения систем с переключениями такого вида предлагается проводить с использованием аппарата общей функции Ляпунова [17, 19].

\section{3. УСТОЙЧИВОСТЬ НУЛЕВОГО РЕШЕНИЯ СИСТЕМ С ПЕРЕКЛЮЧЕНИЯМИ}

В настоящей работе будем рассматривать второй класс систем с переключениями. А именно, системы с неопределенными переключениями. Приведем основные используемые определения.

Определение 1. Нулевое решение системы с переключениями (1) называется устойчивым по Ляпунову, равномерно по переключениям, если для произвольного ее решения $x(t)$ и $\varepsilon>0$ существует такое $\delta(\varepsilon)>0$, что при $t>0$ будет выполняться $|x(t)|<\varepsilon$, лишь только $\max _{-\tau \leq s \leq 0}\{|\phi(s)|\}<\delta(\varepsilon)$.

Определение 2. Нулевое решение системы с переключениями (1) называется асимптотически устойчивым равномерно по переключениям, если оно устойчиво по Ляпунову и $\lim _{t \rightarrow+\infty}|x(t)|=0$.

При исследовании устойчивости одним из основополагающих методов является метод функций Ляпунова. Для систем дифференциальных уравнений суть метода заключалась в существовании положительно определенной функции, полная производная которой в силу системы является неположительной (отрицательно определенной) функцией. Геометрическая интерпретация второго метода Ляпунова состоит в существовании нерасширяющихся и несжимающихся поверхностей уровня $V(x)=c, c>0$ таких, что векторное поле системы направлено внутрь этих поверхностей.

Для разностных систем условие неположительности (отрицательной определенности) полной производной в силу системы заменяется условием неположительности (отрицательной определенности) первой разности в силу системы. Для динамических систем более общего вида, в которых с вычислением производной возникают затруднения, используется понятие «функция убывает вдоль решений». Поскольку будут рассматриваться системы, 
состоящие из подсистем различной структуры, то при получении условий устойчивости будем использовать аппарат «общей функции Ляпунова», который подразумевает существование функции Ляпунова, общей для всех ее подсистем, и убывающей вдоль решений системы с переключениями.

Рассмотрим системы с переключениями, состоящие только из линейных дифференциальных подсистем. Предполагаем, что в точках переключения сохраняется непрерывность, т.е. скачки, обусловленные разностными подсистемами, отсутствуют. Обозначим $S(A)=\left\{S_{i}\left(A_{i}\right), i \in I\right\}$ множество подсистем $S_{i}\left(A_{i}\right)$, которые представляют собой линейные дифференциальные уравнения с постоянными коэффициентами

$$
(A)=\left\{S_{i}\left(A_{i}\right), i \in I\right\}, S_{i}\left(A_{i}\right): \dot{x}(t)=A_{i} x(t), i \in I,
$$

функционирующие на временных промежутках $t \in T_{i}, T_{i}: t_{i-1} \leq t<t_{i}$. В момент времени $t=t_{i}$ происходит переключение на $i+1$ - ю подсистему

$$
S_{i+1}\left(A_{i+1}\right): \dot{x}(t)=A_{i+1} x(t), i \in I .
$$

И функционирование системы с переключениями (2) происходит с выполнением условия непрерывности на следующем промежутке $t \in T_{i+1}, T_{i+1}$ : $t_{i} \leq t<t_{i+1}$. Далее процесс продолжается аналогично. Имеет место следующий результат.

Теорема 1. Чтобы нулевое решение системы с непрерывными переключениями (2), состоящее из линейных подсистем, было асимптотически устойчиво, достаточно, чтобы для ее подсистем существовала общая функиия Ляпунова.

Доказательство. Пусть существует положительно определенная функция $V(x)$, т.е. функция, удовлетворяющая условиям

$$
0<a(|x|) \leq V(x) \leq b(|x|),
$$

где $a(|x|), b(|x|)$ непрерывные функции, удовлетворяющие условиям $a(0)=$ $0, b(0)=0$. И функция $V(x)$ на промежутках времени $T_{i}: t_{i-1} \leq t<t_{i}$ на решениях $x(t)$ подсистем

$$
S_{i}\left(A_{i}\right): \dot{x}(t)=A_{i} x(t)
$$

убывает, а в точках разрыва $t=t_{i}$ сохраняет непрерывность, т.е.

$$
\begin{gathered}
b(|x(0)|) \geq V(x(0))>\ldots>\left.V(x(t))\right|_{t_{i-1}<t \leq t_{i}} \geq V\left(x\left(t_{i}\right)\right)> \\
>\left.V(x(t))\right|_{t_{i}<t \leq t_{i+1}}>a(|x(t)|) .
\end{gathered}
$$

Таким образом для произвольного времени $t>t_{i+1}$ будет выполняться

$$
|x(t)|<a^{-1}(b(x(0)))<\varepsilon .
$$

лишь только $|x(0)|<\delta(\varepsilon)=b^{-1}(a(\varepsilon))$. И нулевое решение системы устойчиво по Ляпунову. Асимптотическая устойчивость следует из строгого убывания функции Ляпунова на временных промежутках $T_{i}: t_{i-1} \leq t<t_{i}$.

Для линейных стационарных дифференциальных систем

$$
\dot{x}(t)=A x(t), t \geq 0
$$


функция Ляпунова обычно строится в виде квадратичной формы $V(x)=$ $x^{T} H x[1,7]$. Матрица $H$ определяется при решении матричного уравнения Ляпунова

$$
A^{T} H+H A=-C
$$

при некоторой положительно определенной матрице $C$. И, если матрица $A$ системы (3) асимптотически устойчива, то при любой положительно определенной матрице $C$ матричное уравнение Ляпунова (4) имеет решением положительно определенную матрицу $H$, а для решений $x(t)$ системы $(3)$ имеет место следующая оценка экспоненциального затухания

$$
\begin{gathered}
|x(t)| \leq \sqrt{\phi(H)}|x(0)| \exp \{-\gamma(H, C) t\}, \\
\phi(H)=\lambda_{\max }(H) / \lambda_{\min }(H), \gamma(H, C)=\lambda_{\min }(C) / 2 \lambda_{\max }(H),
\end{gathered}
$$

где $\lambda_{\min }(\bullet), \lambda_{\max }(\bullet)-$ минимальное и максимальное собственные числа соответствующих матриц. На основании этого утверждения сформулируем более конструктивные условия устойчивости системы с переключениями, состоящей только из линейных дифференциальных подсистем с выполнением условия непрерывности в точках переключения.

Теорема 2. Чтобы нулевое решение системы с переключениями (2), состоящей из линейных дифберенциальных подсистем при сохранении условий непрерывности в точках переключения было асимптотически устойчивым, достаточно, чтобы для всех ее дифференциальных подсистем существовала общая функиия Ляпунова квадратичного вида $V_{0}(x)=x^{T} H_{0} x$, т.е. существовала симметричная, положительно определенная матрица $H$, при которой все матрицы $C_{i}=-A_{i}^{T} H_{0}+H_{0} A_{i}, i \in I$ были положительно определенными. При этом для решения системы с переключениями будет иметь место следующая оценка сходимости

$$
\begin{gathered}
|x(t)| \leq \sqrt{\phi\left(H_{0}\right)}|x(0)| \exp \left\{-\gamma\left(H_{0}\right) t\right\}, \phi\left(H_{0}\right)=\lambda_{\max }\left(H_{0}\right) / \lambda_{\min }\left(H_{0}\right) \\
\gamma\left(H_{0}\right)=\min _{i \in I}\left\{\lambda_{\min }\left(H_{0}, C_{i}\right) / 2 \lambda_{\max }\left(H_{0}\right)\right\} .
\end{gathered}
$$

Доказательство. Следует из предыдущей теоремы и оценок сходимости (5).

Рассмотрим системы с переключениями, которые описаны только линейными разностными подсистемами. Обозначим

$$
S(D)=\left\{S_{j}\left(D_{j}\right), j \in J\right\}, S_{j}\left(D_{j}\right): x(t+1)=D_{j} x(t), j \in J,
$$

систему, которая представляет собой множество разностных подсистем функционирующих на целочисленных промежутках $t=t_{j-1}+1, t_{j-1}+2, \ldots, t_{j}$.

Разностной системой с переключениями $S(D)$ назовем динамическую систему, которая состоит из подсистем разностных уравнений

$$
S_{j}\left(D_{j}\right): x(k+1)=D_{j} x(k), j \in J,
$$

функционирующих на промежутках $T_{j}, j \in J$.

Как и для систем с состоящих только из дифференциальных подсистем с непрерывным переключением, имеет место следующее утверждение. 
Теорема 3. Чтобы нулевое решение системы с переключениями (7), состоящей из линейных разностных подсистем было асимптотически устойчивым, достаточно, чтобы для всех ее разностных подсистем существовала общая функиия Ляпунова $H_{0}$.

Доказательство. Аналогично доказательству предыдущей теоремы.

Для разностных систем (7) функции Ляпунова также имеет вид квадратичной формы $V(x)=x^{T} H x$. Матрица $H$ определяется при решении разностного матричного уравнения Ляпунова

$$
D^{T} H D-H=-G
$$

при некоторой положительно определенной матрице $G$. И, если матрица $D$ асимптотически устойчива (в смысле разностной системы), то при любой положительно определенной матрице $G$ матричное уравнение Ляпунова (8) имеет решением положительно определенную матрицу $H$, а для решений $x(t)$ системы (7) имеет место следующая оценка экспоненциального затухания

$$
\begin{gathered}
|x(k)| \leq \sqrt{\phi(H)}|x(0)|[1-\gamma(H, G)]^{k / 2}, \phi(H)=\lambda_{\max }(H) / \lambda_{\min }(H), \\
\gamma(H, G)=\lambda_{\min }(G) / \lambda_{\max }(H) .
\end{gathered}
$$

Поэтому, по аналогии с теоремой 2 можно сформулировать следующий результат.

Теорема 4. Чтобы нулевое решение системы с переключениями, состоящей из линейных разностных подсистем при было асимптотически устойчивым, достаточно, чтобы для всех ее дифференииальных подсистем существовала общая функиия Ляпунова квадратичного вида $V_{0}(x)=x^{T} H_{0} x$, т.е. существовала симметричная, положительно определенная матрица $H_{0}$, при которой все матрицы $G_{k}=H_{0}-D_{k}^{T} H_{0} D_{k}, k \in K$ были положительно определенными. При этом для решения системы с переключениями будет иметь место следующая оценка сходимости

$$
\begin{gathered}
|x(k)| \leq \sqrt{\phi\left(H_{0}\right)}|x(0)|\left[1-\gamma\left(H_{0}, G_{0}\right)\right]^{k / 2}, \phi\left(H_{0}\right)=\lambda_{\max }\left(H_{0}\right) / \lambda_{\min }\left(H_{0}\right) \\
\gamma\left(H_{0}, G_{0}\right)=\min _{k \in K}\left\{\lambda_{\min }\left(H, G_{k}\right) / 2 \lambda_{\max }\left(H_{0}\right)\right\} .
\end{gathered}
$$

Доказательство. Следует из результатов теоремы 3.

Наконец, рассмотрим системы с переключениями, состоящие из дифференциальных и разностных подсистем, определяющих скачки в моменты переключения. Обозначим через

$$
S(A, D)=\left\{S_{i}\left(A_{i}\right), S_{k}\left(D_{k}\right), i \in I, k \in K\right\}
$$

систему которая состоит из подсистем $S_{i}\left(A_{i}\right)$, представляющих собой системы линейных дифференциальных уравнений (2), функционирующие на промежутках времени $T_{i}: t_{i} \leq t<t_{i+1}$, и подсистем $S_{k}\left(D_{k}\right)$ разностных уравнений (7), определяющих скачки фазовых координат в моменты времени $t=t_{k}$. Для систем с переключениями такого вида имеет место аналогичный результат. 


\section{УСТОЙЧИВОСТЬ НУЛЕВОГО РЕШЕНИЯ СИСТЕМЫ}

Теорема 5. Чтобы нулевое решение системы с переключениями (10), состоящей из линейных дифференииальных подсистем (3), и линейных разностных подсистем (7) было асимптотически устойчивым, достаточно, чтобы для всех ее и дифференииальных, и разностных подсистем существовала общая функиия Ляпунова.

Доказательство. Аналогично доказательству предыдущих теорем 1-3.

Для системы с переключениями, состоящей из линейных дифференциальных и разностных подсистем, общий итоговый итоговый результат имеет следующий вид.

Теорема 6. Чтобы нулевое решение системы с переключениями, состоящей из линейных дифференциальных и разностных подсистем, было асимптотически устойчивым, достаточно, чтобы существовала общая функиия Ляпунова квадратичного вида $V_{0}(x)=x^{T} H_{0} x$, т.е. существовала симметричная, положительно определенная матрича $H_{0}$, при которой матрицы $C_{i}=-A_{i}^{T} H_{0}-H_{0} A_{i}, i \in I, G_{k}=H_{0}-D_{k}^{T} H_{0} D_{k}, k \in K$ были положительно определенными. Причем (если первой подсистемой является дифференииальная), для момента времени $0<t \leq t_{k}$ оценка сходимости имеет следующий вид

$$
|x(t)| \leq \phi\left(H_{0}\right)|x(0)| e^{-\gamma\left(H_{0}\right) t}\left[1-\gamma\left(H_{0}\right)\right]^{n / 2},
$$

где $n$ - число переключений.

\section{ЛИТЕРАТУРА}

1. Barbashin E. A. Lyapunov functions. Moscow: Nauka, 1970. 240 p.

2. Bychkov A. S., Merkuryev M. G. The stability of continuous hybrid automata. Cybernetics and System Analysis. 2007. No. 2. P. 123-128.

3. Bychkov A. S. Sufficient conditions for the exponential stability of the zero stationary state of a hybrid automaton. Mathematical Machines and Systems. 2007. No. 3. P. 168-175.

4. Bychkov A. S. On sufficient stability conditions of hybrid automata with fuzzy switching. Reports of the NAS of Ukraine. 2011. No. 4. P. 7-14.

5. Bychkov A. S. Investigation of the behavior of solutions of differential equations with switchings. National Academy of Sciences of Belarus, Proceedings of the Institute of Mathematics. 2014. No. 1 (22). P. 6.

6. Buslenko N. P. Modeling of complex systems. Moscow: Nauka, 1969. 368 p.

7. Valeev K. G., Finin G. S. Construction of Lyapunov functions. Kiev: Naukova Dumka, 1981. 412 p.

8. Glushkov V. M. Software tools for modeling continuous-discrete systems. Kiev: Naukova Dumka, 1975. 152 p.

9. Emelyanov S. V., Utkin V. I., Taran V. A. et al. Theory of systems with variable structure. Moscow: Nauka, 1970. 592 p.

10. Beetle K. D. Kakhkharov T. E. A study of the stability of logical-dynamic class systems in system design problems. Tashkent: FAN, 1982. 156 p. 
11. Kuzmich O. I. Otsinka zburen rozv'yazkiv non-autonomous systems. News of the Kyiv National University of the Name of Taras Shevchenko. Cybernetics. 2006. No. 7. P. 37-42.

12. Kuzmich O. I., Khusainov D. Ya. Estimation of the dynamics of hybrid systems, which can be described by hybrid systems. News of Kyiv National University of Taras Shevchenko. Cybernetics. 2005. No. 6. P. 45-48.

13. Martynyuk A. A. On the practical stability of hybrid systems. Applied Mechanics. 1989. V. 25. No. 2. P. 101-107.

14. Martynyuk A. A., Slynko V.I. On the stability of linear hybrid mechanical systems with a distributed link. Ukrainian Mathematical Journal. 2008. V. 60. No. 2. P. 204-216.

15. Samoilenko A. M., Perestyuk N. A. Differential equations with impulse action. Kiev: High School, 1987. 286 p.

16. Slynko V. I. On the conditions of stability of motion of linear impulsive systems with delay. Applied Mechanics. 2005. V. 41 (51). No. 6. P. 130-138.

17. Sirenko A. S., Khusainov D. Ya. On the existence of a unified Lyapunov function for linear stationary systems. News of the Kiev National University of Taras Shevchenko. Seriya: Cybernetics. 2013. No. 1 (13). P. 46-51.

18. Filippov A. F. Differential equations with discontinuous right-hand side. Moscow: Nauka, 1985. 255 p.

19. Khusainov D. Ya., Sirenko A. S. On the stability of linear systems with switching. News of the Kyiv National University of Economics Taras Shevchenko. Seriya: Cybernetics. 2014. No. 1 (14). P. 54-60.

Поступила: 04.06.2020 / Принята: 19.06.2020

\title{
СТІЙКІСТЬ НУЛЬОВОГО РОЗВ'ЯЗОКУ СИСТЕМИ 3 ПЕРЕМИКАННЯМ, ЩО СКЛАДАЄТЬСЯ $З$ ЛІНІЙНИХ ПІДСИСТЕМ
}

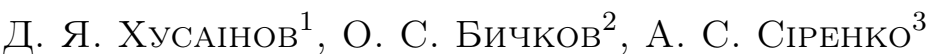

${ }^{1}$ Факультет комп'ютерных наук і кібернетики, Київский національний університет імені Tараса Шевченко, Київ, Україна, E-mail: d.y.khusainov@gmail.com

${ }^{2}$ Факультет інформаційних технологій, Київский національний університет імені Тараса Шевченко, Київ, Україна, E-mail: bos.knu@gmail.com

${ }^{3}$ Інститут міжнародних відносин, Київский національний університет імені Тараса Шевченко, Київ, Україна, E-mail: sandrew@online.ua

АнотАция. У роботі розглядаються питання дослідження стійкості розв'язків динамічних систем з перемиканнями. Отримані достатні умови асимптотичної стійкості нульового розв'язку систем с перемиканнями, що складаються з лінійних диференціальних та різницевих підсистем. Показано, що для асимптотичної стійкості достатньо існування спільної квадратичної функції Ляпунова.

Ключовг СловА: диференціальні рівняння, різницеві рівняння, динамічні системи, система з перемиканнями, функція Ляпунова. 